\title{
TRADUCTION ET EXIL AU XXIEME SIECLE : POUR UNE POETIQUE COLLECTIVE DE RESILIENCE ${ }^{1}$
}

\author{
Ana Paula Coutinho \\ Universidade do Porto. Faculdade de Letras. Departamento de Estudos \\ Portugueses e Românicos. Porto, Portugal.
}

\begin{abstract}
Resumé: Partant du principe que la traduction est un phénomène composite qui dépasse le travail, pourtant fondamental, du traducteur, et qu'il convoque actuellement ce que je propose d'appeler une «poétique collective de résilience», je souligne l'importance de la planification culturelle des traductions, à partir de la sélection faite par les catalogues des éditeurs indépendants, qui travaillent en marge des contingences des grands groupes éditoriaux. Après la présentation des principaux rapports entre l'univers éditorial portugais et l'exil au cours des dernières décennies, j'analyse en particulier le cas des écrivains et des œuvres liés à l'exil du catalogue de la maison d'édition portugaise "Cavalo de Ferro". Mots-clés: Littérature traduite. Exil. Éditeurs. Planification culturelle. Édition Portugal.
\end{abstract}

\section{TRANSLATION AND EXILE IN THE 21TH CENTURY. FOR A COLLECTIVE POETIC OF RESILIENCE}

\begin{abstract}
Assuming that the translation is a composite phenomenon that goes beyond the translator's work, although essential, and currently convenes what I propose to call a "collective poetic of resilience", I stress the importance of cultural planning of translations from the selection made
\end{abstract}

\footnotetext{
${ }^{1}$ Cet article s'insère dans la recherche menée au sein du Programme Stratégique intégré UID/ELT/00500/2013 | POCI-01-0145-FEDER-007339 - « Littérature et frontières de la connaissance : politiques d'inclusion ».
} 
by the catalogues of independent publishers, working at the margins of the contingencies of large publishing groups. After the presentation of the main relationships between the Portuguese editorial universe and the exile over the last decades, I'll analyse, in particular, writers and books related to exil in the catalogue of the Portuguese publishing house "Cavalo de Ferro".

Keywords: Translated literature. Exile. Publishers. Cultural planning. Portugal edition.

\section{Traduction et exil : une association naturelle}

$\mathrm{Au}$ lieu du recours aux métaphores euphoriques (et trop souvent gonflées) de la mobilité pour décrire les phénomènes de la mondialisation et de la société (post-)moderne ${ }^{2}$, il se peut que l'association entre traduction et exil soit plus adéquate pour rendre compte des dynamiques de transfert et de liminarité dont on ne tient pas compte dans les rapports techniques ou dans les statistiques sur la traduction ${ }^{3}$, de l'exil, ou même des deux à la fois. Tout d'abord, on est tenté de considérer que toute œuvre traduite est une œuvre «exilée», puisqu'elle se trouve déplacée de sa langue et de sa culture de départ, assumant une «condition d'étrangère», plus ou moins étrange, dans le système linguistique et culturel d'arrivée. On se penche donc sur les particularités du travail des écrivains ou des traducteurs qui, pour des raisons politiques, religieuses, économiques ou culturelles, ont été amenés (par la force des circonstances ou par choix) à s'exiler et à adopter, directement ou indirectement, une lecture-écriture des cultures «en contrepoint ${ }^{4}$.

Appuyé sur sa propre expérience d'exil, Salman Rushdie, a parlé des écrivains migrants comme d' ${ }^{\prime}$ hommes traduits $»^{5}$. Mais, à vrai

${ }^{2}$ Vd. Anthony Giddens, The Consequences of Modernity. Cambridge: Polity, 1990 et Zygmunt Bauman, Liquid Modernity, Cambridge: Polity, 2000.

${ }^{3}$ Voir, par exemple, celles de Index translationum (http://www.unesco.org/xtrans/bsstatlist. aspx?lg $=0$ ), même s'il s'agit d'un énorme et très important projet

${ }^{4}$ Edward Said, Reflections on exile and other essays. Harvard University Press, 2000, p. 148 .

${ }^{5}$ Salman Rushdie, Imaginary Homelands. London: Granta Books, 1992, p.16- 
dire, cette désignation s'applique à tous les auteurs (post)coloniaux, ainsi qu'à tous ceux qui vivent et écrivent «entre mondes», et qui finissent par adopter une identité littéraire enracinée dans la traduction même, une forme de transitivité entre langues/cultures de départ et langues/cultures d'arrivée - qui vivent en somme, en état de double traduction. D'autre part, n'oublions pas que si l'exil de l'écrivain l'éloigne de son lectorat premier ou de ses lecteurs les plus proches, il se peut aussi qu'il facilite parfois la circulation de son travail, à condition que ses œuvres aient accès à l'univers de la traduction, ne serait-ce qu'à travers la pseudo-traduction ${ }^{6}$, et même si cela suppose de passer par des circuits éditoriaux marginaux ou alternatifs, éventuellement intégrés dans des collections spécifiques de littératures littéralement excentriques.

Par ailleurs, c'est à la suite de leur émigration ou de leur exil que certaines personnes sont devenues des traducteurs, notamment à partir de langues dont il n'existait pas, ou très peu, de traductions directes dans les pays d'accueil. Certains autres, notamment ceux issus des diverses diasporas et culturellement hybrides, retournent symboliquement à la langue et au monde de leurs origines à travers la traduction - que ce soit l'auto-traduction ou la traduction par autrui. Qu'il s'agisse de traduction interlinguistique ou de transferts culturels ${ }^{7}$, nous sommes toujours face à une construction ou déconstruction des images autour des cultures d'origine et/ou d'accueil, propres à ceux qui observent à distance - physique ou mentale. C'est pourquoi ces auteurs vivent souvent en état de double exil, pétris des «douleurs de l'arrachement» ${ }^{8}$. D'une part, ils sont en marge de la langue et de la culture du pays où ils sont installés, puisque ce qu'ils écrivent, leurs références sociales et culturelles n'intéressent qu'une petite minorité de lecteurs; d'autre part, même

\footnotetext{
${ }^{6}$ Ce qui s'est passé, par exemple, au début des années 90, avec les deux premiers romans d'Andreï Makine, écrivain français d'origine russe, présentés comme des traductions afin d'intéresser davantage le public français.

${ }^{7}$ Vd. Michel Espagne, «La notion de transfert culturel», Revue Sciences/Lettres, 1, 2013 en ligne: https://rsl.revues.org/219 [consulté le 30 octobre 2015].

${ }^{8}$ Cf. Camille de Toledo, Le Hêtre et le Bouleau, Paris : Seuil, 2009, p.200.
} 
quand ils sont reconnus ou promus à travers, par exemple, des prix littéraires, il arrive que ce succès provienne d'une attente socioculturelle ou d'une pression médiatique imposant des quotas de la «différence», voire de l'«exotique». Et pourtant, du point de vue esthétique, ce sont souvent les auteurs et les œuvres en exil, (sur)vivant dans les marges sociales, culturelles et linguistiques, qui contribuent le plus à la déterritorialisation du langage et du récit - au sens de force intrinsèquement politique d'une «littérature mineure», pour utiliser le fameux concept que Deleuze et Guattari ont développé à partir de Kafka9 .

\section{En amont et en aval de la traduction: le rôle déterritorialisant des éditeurs}

S'il est vrai que les différentes vagues dans les études de traduction au cours du XXème siècle ont été décisives pour la légitimation et pour la valorisation socioculturelles de la traduction et si, du coup, il est irréfutable qu'elles ont affranchi l'invisibilité presque systématique du traducteur ${ }^{10}$, il ne faut pas oublier que la littérature traduite est un processus de réécriture qui ne commence ni ne s'achève dans l'acte de traduire par un individu, comme le faisait déjà noter André Lefevere, en parlant de "patronage » comme effet du pouvoir des différents agents et institutions gravitant autour de la traduction ${ }^{11}$. Plus récemment, venus du côté de la sociologie de la culture, Johan Heilbron et Gisèle Sapiro ont fait remarquer que l'étude des «pratiques de la traduction littéraire exige une double rupture, aussi bien avec l'approche herméneutique du texte et de ses transmutations qu'avec l'analyse

\footnotetext{
${ }^{9}$ Cf. Gilles Deleuze ; Felix Guattari, Kafka. Pour une littérature mineure. Paris : Minuit, 1975.

${ }^{10}$ Lawrence Venuti, Scandals of Translation. Towards an Ethics of Difference. London: Routledge, 1998.

${ }^{11}$ André Lefevere, Translation, Rewriting, and the Manipulation of Literary Fame. London/ New York: Routledge, 2002, p.15.
} 
purement économique des échanges transnationaux et des transferts culturels» ${ }^{12}$. C'est aussi à ce nouveau paradigme d' «articulation» dans la «chaîne de la traduction» qu'appelait, en 2012, l'état des lieux de Transeuropéennes et de la Fondation Anna Lindh ${ }^{13}$ consacré à la traduction dans la région euro-méditerranéenne - où l'on a beaucoup insisté sur l'importance de «l'ensemble des acteurs engagés dans le processus de circulation des ouvrages traduits: auteurs, traducteurs, éditeurs, libraires, bibliothécaires, critiques, organismes de soutien» ${ }^{14}$. De cela il ressort que l'on peut parler de la traduction littéraire comme d'une action ou d'une poiesis collective, qui mise sur les différents enjeux de la médiation entre le pôle de départ et celui de la langue et culture d'accueil.

À ce propos, concentrons-nous sur la figure de l'éditeur et, plus généralement, de la maison d'édition, qu'on n'avait pas vraiment l'habitude de prendre en considération, mais qui jouent un rôle fondamental dans la vie littéraire. Du reste, il s'agit d'une importance toujours croissante depuis le XIXème siècle : des éditeurs-libraires, bibliophiles fiers, qui vivent leur activité comme une forme de mécénat et de militance culturelle, aux éditions-entreprises, avec leurs organigrammes d'une complexité grandissante comprenant des responsables pour chaque section des catalogues, des comités de lecture, des experts de marketing, bref toute une série d'intervenants. De surcroît, quand il s'agit d'éditer des ouvrages étrangers, ce long processus représente une forme d'importation, un transfert et, implicitement, la manipulation de deux patrimoines intellectuels et artistiques. De fait, en plus de ses répercussions au niveau de l'internationalisation d'un auteur ou d'une littérature, la traduction est censée élargir, légitimer et/

\footnotetext{
${ }^{12}$ John Heilbron / Gisèle Sapiro, «La traduction littéraire, un objet sociologique», Actes de la recherche en sciences sociales, $\mathrm{n}^{0} 144$ (2002), Paris : Seuil, p.3.

${ }^{13} \mathrm{Cf}$. http://www.transeuropeennes.eu/fr/articles/342/Conclusions_et_recommandations

${ }^{14}$ Idem, s/p. D'autres projets comme La Société européenne des Auteurs (http://www.seua. org/fr/) ou Schwob. The World's Best Unknown Books (http://en.schwob-books.eu/) essaient aussi de combler des lacunes dans la traduction de littérature et, en générale, de la pensée artistique et scientifique, misant sur partenariat des différents agents de ce long et formateur processus culturel.
} 
ou flexibiliser le système linguistique et culturel cible, développant ainsi les ressources esthétiques et le fonds de pensée d'une certaine langue et de sa littérature (ou de ses littératures, pour les cas des langues internationales, comme le français, le portugais ou l'anglais). Les critères que les éditeurs (et derrière, ou à leur côté, les directeurs de collection, des consulteurs, des traducteurs...) utilisent pour constituer un catalogue s'avèrent donc tout à fait décisifs, i. e. modélisants. Ce sont eux qui dictent la sélection d'auteurs étrangers et de leurs textes, en fonction des besoins, des intérêts ou des caractéristiques du réseau, par exemple, en fonction des catalogues concurrents, des valeurs sur le marché international des livres et des négociations avec les auteurs étrangers ou leurs agents littéraires ${ }^{15}$. Ce sont aussi les éditeurs qui choisissent tous les intervenants dans ce travail de médiation, des traducteurs aux réviseurs en passant par ceux qui conçoivent les couvertures ou qui travaillent à la distribution et diffusion des livres. Sans une articulation efficace entre toutes ces étapes et tous ces agents, la littérature traduite risquerait de ne pas arriver au public en général et de n'être ni lue ni retenue par les lecteurs professionnels (critiques, éditeurs, universitaires, acteurs...), ainsi que par ces autres lecteurs particulièrement importants que sont les écrivains, d'autant plus que toute histoire de la littérature nous montre que ce sont les écrivains qui, finalement, assurent les effets les plus importants et les plus durables d'une traduction littéraire. À ce propos, il suffit de penser à l'apport syncrétique de l'«esthétique de la réception», depuis l'essai fondateur de Hans Robert Jauss en 1967, L'histoire littéraire comme provocation, surtout quand cette esthétique de la réception est parvenue à associer herméneutique littéraire et lecteurs empiriques déterminants comme c'est le cas des écrivains. Or, souvent, non seulement les écrivains ont contacté avec les littératures étrangères à travers des traductions, mais ils ont été aussi des traducteurs, surtout en début de carrière.

15 'A propos, voir Ann Steiner, «World Literature and the Book Market», The Routledge Companion to World Literature, Edited by Theo D'Haen, David Damrosch and Djelal Kadir, London and New York: Routledge, 2012, p. 316-324.

Cad. Trad., Florianópolis, v. 38, n $^{0}$ 1, p. 181-196, jan-abr, 2018 
Sans sous-estimer aucunement l'autonomie relative de la littérature et sans oublier quelques spécificités de la «condition littéraire» ${ }^{16}$, qui recouvre aussi la traduction et qui ne coïncide pas exactement avec les modèles d'observation et de catégorisation habituels en sociologie, il est donc important de concevoir la traduction littéraire comme un processus collectif, et de reconnaître le travail en fonction de la visibilité de l'éditeur, ainsi que celui des autres agents qui participent à l'inclusion, toujours déterritorialisante, des auteurs étrangers dans un certain «polysystème» ${ }^{17}$.

Ce n'est peut-être pas par hasard qu'il y a des éditeurs (et, dans certains cas, des libraires également) qui furent ou sont des exilés, i. e, éloignés de leur langue e/ou culture d'origine. $\mathrm{Au}$ Portugal, à part l'importante intervention d'éditeurs/libraires d'origine française comme Pedro Faure, fondateur au XVIIIème siècle de la Librairie Bertrand qui est devenue aussi une maison d'édition et reste aujourd'hui encore le plus grand réseau de librairies dans le pays ${ }^{18}$, ou comme «Aillaud\&Lellos», il y a eu aussi une femme-éditeur d'origine danoise très importante, Snu Abecassis, qui a vécu en Suède et aux États-Unis avant de s'installer au Portugal dans les années 60 du siècle dernier, où elle a fondé la maison d'édition «Publicações Dom Quixote», avec une très importante collection de littérature traduite, qui existe toujours sous le nom de «Ficção Universal» [Fiction Universelle»]. Cette maison d'édition apporta une vraie note de fraîcheur dans l'ambiance lourdement grise imposée par la dictature à l'époque. Snu Abecassis a d'ailleurs eu quelques problèmes avec la police politique de l'époque (la PIDE) pour avoir invité à Lisbonne le poète soviétique Yevgny Yevtuchenko, et pour avoir édité, en 1971, un livre de poèmes de Maria Teresa Horta - une des très célèbres femmes-écrivaines nommées les trois Maries (Maria Isabel

\footnotetext{
${ }^{16}$ Bernard, Lahire, La Condition Littéraire. Paris : La Découverte, 2006.

17 Itamar, Even-Zohar, "The Position of Translated Literature within the Literary Polysystem», Poetics Today 11:1 (1990), 45-51.

${ }^{18}$ Associée entre-temps au plus grand groupe éditorial au Portugal: «Porto Editora»
} 
Barreno, Maria Teresa Horta et Maria Velho da Costa), auteures de Novas Cartas Portuguesas, une œuvre à six mains, tout à fait originale et marquante. Bien que ce livre ait été immédiatement retiré de circulation et détruit par la censure, il représente un cas tout à fait singulier d'extraduction de littérature portugaise et une référence pour les études féministes et pour la créativité issue du discours littéraire d'une façon générale ${ }^{19}$. En dehors du Portugal, il y a aussi des éditeurs qui, à la suite d'une expérience d'exil ou d'expatriation, se sont lancés dans l'aventure de la traduction de la littérature (ou des littératures) de langue portugaise. C'est le cas de Michel Chandeigne qui, de retour en France après avoir vécu quelques années à Lisbonne, a commencé par fonder, en 1986 à Paris, la Librairie Portugaise et Brésilienne, et un peu plus tard (avec Anne Lima), en 1992, les Éditions Chandeigne, qui privilégient toujours la traduction d'auteurs de langue portugaise. Michel Chandeigne traduit lui-même des poètes comme Fernando Pessoa, António Ramos Rosa ou Mário de Sá-Carneiro. Consacrés plutôt à l'univers de la diaspora portugaise du XXème siècle, deux autres éditeurs - João David Rosa e João Heitor - ont aussi associé leur expérience exilique à des projets éditoriaux. João Heitor, qui vit en France depuis les années 70, a commencé avec une librairie - «La librairie lusophone» - en plein Quartier Latin, et plus récemment il a également fondé les «éditions lusophones». Bien que la traduction littéraire ne soit pas au cœur de ce projet, il s'agit d'une forme de présence de la culture portugaise en France qui se propose d'établir des ponts avec les nouvelles générations de français liés au monde lusophone. En ce qui concerne João David Rosa, il fut, au début des années 80, un autodidacte pionnier, qui avec très peu de moyens, a édité, depuis la Suisse où il était immigré, la revue Peregrinação, à laquelle il a associé aussi une collection d'ouvrages liés à la diaspora portugaise ${ }^{20}$. De même,

\footnotetext{
${ }^{19}$ Vd. Novas Cartas Portuguesas - Entre Portugal e o Mundo, Organização de Ana Luísa Amaral e Marinela Freitas. Lisboa: Publicações Dom Quixote, 2014.

${ }^{20} \mathrm{Vd}$. Ana Paula Coutinho Mendes, Lentes Bifocais. Representações da Diáspora Portuguesa do Século XX. Porto: Afrontamento, 2009, 15-29.
} 
et actuellement, «Oxalá Editora ${ }^{21}$, maison d'édition siègée à Dortmund (Allemagne), a comme but est d'intervenir directement au niveau de la diaspora du monde lusophone.

\section{Editeurs doublement périphériques à l’ère de la mondialisation: le cas de «Cavalo de Ferro» au Portugal}

Approfondissant le modèle théorique de Pierre Bourdieu concernant le «champ littéraire» et «les règles de l'art», les travaux de Pascale Casanova ${ }^{22}$ et de Gisèle Sapiro ${ }^{23}$, menés avec exhaustivité à partir du contexte français, sont venus démontrer clairement - ou plutôt confirmer - que les échanges entre littératures sont très hiérarchisés et que ce type de rapport est à la fois cause et conséquence d'un commerce fort asymétrique au niveau des traductions. Nonobstant, il faut souligner que l'opposition entre dominants et dominés ne reproduit pas toujours ce qui se passe du côté du sociopolitique ${ }^{24}$. D'une part, les résultats de la recherche sur le marché de la traduction en France à l'ère de la mondialisation, dirigée par Gisèle Sapiro au sein du CNRS, démontrent que l'impression générale de la suprématie grandissante des traductions à partir de l'anglais est fondée, et prouvent, en même temps, que «la littérature est le secteur au taux de traduction le plus élevé, et celui où la diversité des langues d'origine est la plus grande» ${ }^{25}$. Néanmoins, les importateurs (éditeurs, traducteurs, critiques) doivent être très persistants pour résister au «Mclanguage of a

\footnotetext{
${ }^{21}$ Vd. http://oxalaeditora.de/Portugu\%C3\%AAs/editora.html.

${ }_{22}^{2}$ Vd. Pascale Casanova, La République mondiale des lettres. Paris : Coll. Points, 2008; Pascale Casanova, La langue mondiale. Traduction et domination. Paris : Seuil, 2015

${ }^{23} \mathrm{Vd}$. Gisèle Sapiro, Translatio. Le marché de la traduction en France à l'heure de la mondialisation. Paris : Éditions du CNRS, 2009

${ }^{24}$ Pascale Casanova parle du «miracle irlandais», et l'on pourrait ajouter aussi les cas de Kafka ou de Pessoa.

${ }^{25}$ Cf. Gisèle Sapiro, Translatio. Le marché de la traduction en France à l'heure de la mondialisation. Paris : Éditions du CNRS, 2009, p.145.
} 
globalized «Mcworld» ${ }^{26}$, et parvenir à faire entrer, i. e. à faire découvrir, un auteur ou une littérature d'une langue périphérique ou semi-périphérique ${ }^{27}$. Dans le chapitre «Globalisation» de son essai de 1998, Scandals of Translation. Towards an Ethic of Difference, Lawrence Venuti s'était déjà penché sur la traduction comme acte de résistance pendant le colonialisme tout en soulignant l'ambivalence de son rôle ${ }^{28}$, ainsi que les aspects négatifs de l'importation des best-sellers anglo-américains dans les pays émergents $^{29}$, (t pas uniquement dans ceux-là, pourrait-on ajouter. Comme cet essayiste le montrait, ces traductions résultent d'une action concertée de groupes multinationaux, englobant des maisons d'édition, des distributeurs et des agences de marketing, dont les effets fonctionnent comme une dissémination/imposition culturelle semblable à celle qu'a représenté le colonialisme européen ${ }^{30}$. C'est ce que dénonce aussi le tout récent essai de Pascale Casanova, intitulé La langue mondiale. Traduction et domination: «Le propre de la langue mondiale, en effet, c'est de se répandre plus vite que les autres (notamment à travers les «opérations de traduction») et d'imposer les catégories de pensée qui lui sont attachées (si l'on suit l'hypothèse Sapir-Whorf) à ceux qui la maîtrisent (...)» ${ }^{31}$. C'est justement contre cette hégémonie linguistique ${ }^{32}$, mais qui est aussi une hégémonie culturelle et de la pensée en général, que s'est insurgé le linguiste Claude Hagège ${ }^{33}$, car une telle domination exige plus que de la résistance, une attitude de résilience - au sens

\footnotetext{
${ }^{26}$ Cf. Mary Snell-Horby, The Turns of Translation Studies. Amsterdam/Philadephia: John Benjamins Publishing Company, 2006, p.144.

${ }^{27}$ Cf. Gisèle Sapiro, Translatio. op.cit., 2009, p.153.

${ }^{28}$ Lawrence Venuti, Scandals of Translation. Towards an Ethics of Difference, op.cit, p. 170.

${ }^{29}$ Idem, p.162.

${ }^{30}$ Idem, p. 165.

${ }^{31}$ Cf. Pascale Casanova, La langue mondiale. Traduction et domination, Paris : Seuil, 2015, p.129.

${ }^{32}$ Une hégémonie à laquelle contribue aussi - au Portugal comme ailleurs - une exigence de formation en langues étrangères assez restreinte, souvent limitée à une seule langue...

${ }^{33}$ Vd. Claude Hagége, Halte à la mort des langues. Paris : Éditions Odile Jacob, 2001 ; Claude Hagège, Contre la pensée unique. Paris: Éditions Odile Jacob, 2012.
} 
d'une capacité à se refaire après un choc ${ }^{34}$ - de la part des maisons d'édition autonomes ou, du moins, plus indépendantes en regard du monopole des grands groupes éditoriaux, infiltrés un peu partout, liés aux réseaux internationaux et souvent insondables du capital.

À titre d'exemple d'éditeurs qui fonctionnent, autant que possible, à l'écart de cette logique hégémonique, je présenterai ici brièvement «Cavalo de Ferro», une maison d'édition qui a commencé son activité en 2003, avec l'objectif d' «offrir un spectre de littératures, à l'époque non disponibles, au public portugais, tout en les traduisant à partir des langues originales» ${ }^{35}$, et qui est parvenue à subsister grâce à des projets menés avec des partenariats divers $^{36}$, alors que d'autres petits éditeurs indépendants, qui voulaient eux aussi fonctionner comme alternative aux catalogues des grands groupes éditoriaux, ne résistent pas longtemps ${ }^{37} \ldots$

Dans la présentation en ligne de «Cavalo de Ferro» - qui comprend l'adaptation signée par un des éditeurs du poème de e. e. cummings, 'No thanks' -, on peut lire: «La vraie littérature n'est pas celle qui nous distrait, mais plutôt celle qui nous concentre». Ensuite, on apprend aussi que «Cavalo de Ferro» est une maison d'édition spécialisée en traduction et publication de littérature étrangère au Portugal, ayant édité «entre classiques et contemporains, entre auteurs consacrés et auteurs émergeants, entre poésie et prose, quelques uns des plus importants auteurs

\footnotetext{
${ }_{34}$ J'adopte cette notion, qui vient de la psychologie, pour désigner ici la capacité de reconstruction des agents de la traduction littéraire, après l'impact provoqué par notre ère de marchandise globale, touchant (aussi) le domaine de la littérature.

${ }^{35}$ Déclarations de Hugo Freitas Xavier, un des éditeurs de « Cavalo de Ferro » (Xavier 2006: 88). Pour les langues moins connues, ils ont fait appel à des traducteurs non spécialisés, dont le travail a été accompagné par des réviseurs littéraires et par tout le département éditorial.

${ }^{36}$ Ces projets éditoriaux ont été réalisés en collaboration avec des groupes de la communication sociale (presse hebdomadaire) et avec d'autres entreprises, comprenant des livres, des fascicules, des CD-Audio et des DVD, pour être diffusés au-delà du circuit des librairies.

${ }^{37}$ Notamment, par exemple, la toute petite maison d'édition "Ahab", qui siège à Porto et qui a débuté son activité en 2009, sous les meilleurs auspices (elle a même reçu le prix de maison d'édition-révélation), misant sur la traduction d'auteurs et d'ouvrages littéraires alternatifs ou ex-centriques. Malheureusement, son activité s'est évanouie après la publication d'un nombre de titres très réduit.
}

Cad. Trad., Florianópolis, v. 38, n $^{0}$ 1, p. 181-196, jan-abr, 2018 
de la littérature internationale», dont beaucoup qui n'avaient pas encore été édités au Portugal, comme les nord-américains Bernard Malamud (1914-1996) et Shirley Jackson, les islandais Haldor Laxness (Prix Nobel 1955) et Thor Vilhjálmsson; l'irlandais Flann O'Brien, le nobel norvégien Eyvind Johnson (1974), l'écrivain belge Yves Namur, l'italienne Romana Petri, ou l'urugayen Horacio Quiroga, le soudanais Al-Tayyeb Salih, ou l'argentin installé à Barcelone, Rodrigo Fresán. Outre leur appartenance à des langues périphériques, telles que l'islandais, le serbo-croate, le norvégien, le hongrois, le polonais ou le japonais, bon nombre d'auteurs publiés par «Cavalo de Ferro» sont aussi liés à différentes expériences d'exil comme l'auteur yougoslave Ivo Andric, le juif séfarade d'origine bulgare Elias Canetti, le portugais Ferreira de Castro, immigré au Brésil, l'écrivain argentin, né en Belgique et exilé à Paris, Julio Cortázar, l'écrivain norvégien Knut Hamsun, qui a longtemps vécu aux États- Unis, l'écrivaine Dubravka Ugrešić qui, ayant quitté la Croatie, vit désormais au Pays-Bas, le grec Pakos Karnezis installé à Londres, l'écrivain Wladimir Kaminer, né en ex-Union Soviétique, mais immigré en Allemagne depuis 1990, la hongroise Agota Kristof qui a fui son pays natal dans les années 50 pour vivre en Suisse jusqu'à sa mort en 2011, Vernon Lee, pseudonyme de l'écrivaine, historienne et philosophe Violet Paget (1856-1935), née en France, éduquée en Allemagne, et ayant vécu une grande partie de sa vie en Italie, le poète polonais Czeslaw Milosz, exilé tout d'abord à Paris puis aux Etats-Unis... Ceci dit, on ne saurait en conclure que «Cavalo de Ferro» offre au lecteur portugais ou de langue portugaise une liste d'auteurs complètement en marge de la «République mondiale des Lettres», pour utiliser la très suggestive expression de Pascale Casanova. Quelques-uns des auteurs cités ont même reçu le Prix Nobel de la Littérature... Toutefois, on comprend aisément que le catalogue de «Cavalo de Ferro» mise sur l'inclusion dans le polysystème littéraire portugais d'auteurs et de langues périphériques ou semi-périphériques, avec une démarche très significative au niveau de la reconnaissance de 
la diversité européenne ${ }^{38}$. Bien que ce ne soit pas un cas unique dans le contexte éditorial portugais, le catalogue de «Cavalo de Ferro» représente un élargissement/renouvellement au Portugal du répertoire de littérature étrangère, en particulier dans le domaine de la fiction. «Cavalo de Ferro» a opté pour une logique de production restreinte et une diffusion à long terme, ce qui représente donc un modus operandi alternatif par rapport à la posture dominante guidée par la bourse de valeurs des «best-sellers» fugaces du circuit international, et dont les droits de traduction sont d'habitude acquis et transmis entre grands groupes éditoriaux.

Bref, s'il existe, comme je l'ai souligné au début, un rapport très étroit entre «traduction et exil» ou entre «exil et traduction», tous deux marqués par la complexité et l'ambivalence de la quête d'un espace propre, il s'avère de plus en plus urgent d'associer à l'exil des auteurs et des textes traduits, la résilience de la part de tous ceux (en particulier les éditeurs indépendants et leurs collaborateurs) qui sont les premiers à contribuer à ce qu'un auteur déplacé, sinon même expulsé, de son espace et de sa langue d'origine, puisse être accueilli au sein d'une autre littérature. L'articulation entre tous les intermédiaires de la littérature traduite dans un système particulier devrait du reste intégrer les objectifs généraux d'une planification culturelle $^{39}$, qui pour autant ne doit pas signifier des prescriptions centralisatrices ou des impositions découlant des agendas politiques.

L'implication de tous les agents concernés par la dynamique de la traduction littéraire (aussi bien dans les littératures de départ que dans la littérature-cible) aboutirait donc à ce que je propose de nommer une "poétique collective de résilience», une action commune et intégrée d'éditeurs, traducteurs, critiques, professeurs... cherchant à assurer la diversité culturelle, l'ouverture d'un système littéraire à différentes voix/voies étrangères.

\footnotetext{
${ }^{38}$ Voir l'élargissement de son catalogue littéraire en 2004, à la suite de l'entrée de 10 pays dans l'Union Européenne, avec une collection thématique: 10 livres pour une Nouvelle Europe.

${ }^{39} \mathrm{Cf}$. Gideon Toury, «Enhancing cultural changes by means of fictitious translations», Translation and Cultural Change, edited by Eva Hung. Benjamin Translation Library, 2005, p. 9.
} 
Si cette poétique collective est incapable de résoudre tous les problèmes structurels de notre époque de migrations et d'exils en masse, elle pourrait du moins empêcher que la littérature soit transformée en un espace de plus en plus homogène, où ceux qui sont loin de leurs origines, ou à la marge du pouvoir économique ou symbolique de légitimation, se trouvent du même coup condamnés à l'ostracisme d'une catégorie exogène, soumis à l'évanescence dans le silence; destinés à la non-traduction, à la non-publication, à la non-lecture, à l'absence de réception critique... Bref, un destin d'exclusion recouvrant une autre forme d'exil, qui finit par toucher tout le monde, puisqu'il contribue à l'épanouissement d'une autosuffisance ignorante, même quand elle est masquée d'un soidisant cosmopolitisme.

\section{Références}

Bauman, Zygmunt. Liquid Modernity. Cambridge: Polity, 2000.

Casanova, Pascale. La République mondiale des lettres. Paris : Coll. Points, 2008

Casanova, Pascale. La langue mondiale. Traduction et domination. Paris : Seuil, 2015.

Deleuze, Gilles; Guattari, Félix . Kafka. Pour une littérature mineure. Paris : Minuit, 1975.

Espagne, Michel. «La notion de transfert culturel», Revue Sciences/Lettres, 1, 2013 en ligne: https://rsl.revues.org/219 [consulté le 30 octobre 2015]

Even-Zohar, Itamar. «The Position of Translated Literature within the. Literary Polysystem», Poetics Today 11.1 (1990): 45-51. 
Giddens, Anthony. The Consequences of Modernity. Cambridge: Polity, 1990.

Hagège, Claude. Halte à la mort des langues. Paris : Éditions Odile Jacob, 2001.

Hagège, Claude. Contre la pensée unique. Paris: Éditions Odile Jacob, 2012.

Heilbron, Johan/Sapiro, Gisèle. «La traduction littéraire, un objet sociologique», Actes de la recherche en sciences sociales. Paris: Seuil 144 (2002): 3-5.

Lahire, Bernard. La Condition Littéraire. Paris : La Découverte, 2006.

Lefevere, André. Translation, Rewriting, and the Manipulation of Literary Fame. London/New York: Routledge, 2002.

Mendes, Ana Paula Coutinho. Lentes Bifocais. Representações da Diáspora Portuguesa do Século XX. Porto: Afrontamento, 2009.

Novas Cartas Portuguesas - Entre Portugal e o Mundo, Organização de Ana Luísa Amaral e Marinela Freitas. Lisboa: Publicações Dom Quixote, 2014.

Rushdie, Salman. Imaginary Homelands. London: Granta Books, 2001.

Said, Edward. Reflections on exile and other essays. Harvard University Press, 2000.

Sapiro, Gisèle. Translatio. Le marché de la traduction en France à l'heure de la mondialisation. Paris : Éditions du CNRS, 2009.

Snell-Horby, Mary. The Turns of Translation Studies. Amsterdam/Philadephia: John Benjamins Publishing Company, 2006.

Steiner, Ann. «World Literature and the Book Market», The Routledge Companion to World Literature, Edited by Theo D'Haen, David Damrosch and Djelal Kadir, London and New York: Routledge (2012): 316-324. 
Toledo, Camille de. Le Hêtre et le Bouleau. Essai sur la Tristesse Européenne, Paris: Seuil.

Toury, Gideon. «Enhancing cultural changes by means of fictitious translations», Translation and Cultural Change, edited by Eva Hung. Benjamin Translation Library, 2005.

Venuti, Lawrence. Scandals of Translation. Towards an Ethics of Difference. London: Routledge, 1998.

Xavier, Hugo Freitas. "Notas sobre a Tradução Literária em Portugal, sua evolução e quadro actual». Babilónia, . Lisboa: Universidade Lusófona 4 (2006): 85-90.

Recebido em: 22/08/2017

Aceito em: 13/10/2017

Publicado em janeiro de 2018

Ana Paula Coutinho é Professora Associada com Agregação do Departamento de Estudos Portugueses e Românicos da Faculdade de Letras da Universidade do Porto, onde lecciona nas áreas da Literatura Comparada e dos Estudos Franceses. Nos últimos anos, tem desenvolvido particular investigação no domínio das interculturalidades, nomeadamente das representações literárias, culturais e artísticas das migrações e exílio. Actual Coordenadora Científica Geral da I\&D - Instituto de Literatura Comparada Margarida Losa, onde também coordena a enciclopédia digital Ulyssei@s Escritores e outros criadoresem deslocação.... Porto, Portugal. E-mail: amendes@letras.up.pt 